\section{Increased meningococcal carrier rate after tonsillectomy}

The meningococcal carrier state induces bactericidal serum antibodies which correlate with protection against meningococcal disease. ${ }^{1}$ Colonisation of the nasopharynx by Neisseria meningitidis, however, also precedes invasive meningococcal disease. Factors determining the outcome of nasopharyngeal meningococcal colonisation are not known. As the palatine tonsils are the largest lymphoid organs in that area, we studied the effect of tonsillectomy on the meningococcal carrier rate.

\section{Patients, methods, and results}

Two specimens of throat flora (specimens 1 and 2) were collected three months apart from 160 patients admitted to hospital for tonsillectomy and from 107 healthy controls. All first specimens from the patients and first and second specimens from the controls were collected by us and incubated immediately; the second specimens from the patients were collected by their own doctors and sent to us by mail. Only specimens with a transport time shorter than 24 hours were included in the study. All specimens were cultured on a selective medium and meningococci identified and serogrouped according to standard procedures.

For ease of reporting we refer to meningococcal carriers as $\mathrm{M}+$ and noncarriers as $M-$. Statistical analysis was by Fisher's exact test.

Of the 160 patients, 85 were excluded; in three tonsillectomy was cancelled, 41 did not attend for collection of their second specimen, and in 41 cases the transport time of specimen 2 exceeded 24 hours. The mean age of the remaining 75 patients ( 41 female) was 16.9 years. The mean interval between collections of specimens 1 and 2 was 100 days.

Of the 107 controls, 68 attended for collection of specimen 2. Their mean age was 17.9 years, and 49 were female. The mean interval between collections of the two specimens was 96 days.

Thirteen patients and six controls were initially $M+$, but during the observation period three patients and two controls became $M_{-}$- (table).

Number of meningococcal carriers $(M+)$ and non-meningococcal carriers $(M-)$ among 75 patients before (specimen 1) and three months after tonsillectomy (specimen 2) and among 68 healthy controls sampled at similar intervals

\begin{tabular}{|c|c|c|c|c|c|c|}
\hline \multirow{3}{*}{$\begin{array}{l}\text { Meningococcal } \\
\text { carrier state } \\
\text { as determined } \\
\text { from specimen } 1\end{array}$} & \multicolumn{3}{|c|}{ Patients } & \multicolumn{3}{|c|}{ Controls } \\
\hline & \multicolumn{2}{|c|}{ Specimen 2} & \multirow{2}{*}{ Total } & \multicolumn{2}{|c|}{ Specimen 2} & \multirow{2}{*}{ Total } \\
\hline & $M+$ & $M-$ & & $M+$ & $\mathbf{M}-$ & \\
\hline $\begin{array}{l}M+ \\
M-\end{array}$ & $\begin{array}{l}10 \\
15\end{array}$ & $\begin{array}{r}3 \\
47\end{array}$ & $\begin{array}{l}13 \\
62\end{array}$ & $\begin{array}{l}4 \\
0\end{array}$ & $\begin{array}{r}2 \\
62\end{array}$ & $\begin{array}{r}6 \\
62\end{array}$ \\
\hline Total & 25 & 50 & 75 & 4 & 64 & 68 \\
\hline
\end{tabular}

By contrast, 15 of the $62 \mathrm{M}$ - patients but none of the $62 \mathrm{M}$ - controls converted to $\mathrm{M}+(\mathrm{p}<0.00001)$. The number of patients converting from $M$ - to $M+$ was significantly higher than the number converting from $M+$ to $M-(p<0.0001)$.

Twenty of the 25 strains of meningococci isolated from specimen 2 from the patients and three of the four strains from the controls were encapsulated; group B meningococcus was more frequently isolated than other groups.

\section{Comment}

A significantly higher number of patients with tonsillectomy than controls became meningococcal carriers during the observation period $(\mathrm{p}<0.00001)$. Factors responsible for the difference are not clear. It is unlikely that differences between the two groups were accounted for by differences in the collection of specimens, because specimen 2 from the patients was subject to a transportation time of 18-24 hours before plating; if anything, this would be expected to decrease the number of viable meningococci. Hence removal of the tonsils presumably caused a local change in the mucous membranes, favouring their colonisation by $N$ meningitidis.

It seems unlikely that changes in the microflora of the pharynx after tonsillectomy explain the increased meningococcal carrier rate because tonsillectomy apparently recreates the normal microflora of this area. ${ }^{2}$ The possibility that immunological factors were implicated in the conversion from $M-$ to $M+$ carrier state deserves consideration. Probably the most important immunological constituent of the mucus bathing these surfaces is secretory IgA, which seems to inhibit the adherence of micro-organisms to the mucosal epithelial cells.
A significant but transient decrease in the relative concentration of salivary IgA has been observed in the first few months after tonsillectomy. ${ }^{3}$ In addition, reduced concentrations of specific antibodies in nasopharyngeal mucus have been observed after tonsillectomy. ${ }^{4}$

Many questions raised by our study remain to be answered. Does tonsillectomy lower the amount of specific antimeningococcal IgA of the nasopharyngeal mucus? Does tonsillectomy decrease the host's resistance to invasive meningococcal disease?

We thank Dr Odd Aalen, of the University of Oslo, for the statistical analyses.

1 Reller LB, MacGregor RR, Beaty HN. Bactericidal antibody after colonization with Neiserria meningitidis. I Infect Dis 1973;127:56-62.

2 Sprinkle PM, Veltri R. Recurrent adenotonsillitis: a new concept. Laryngoscope 1976;86:58-63.

${ }^{3}$ Jeschke R, Strøder J. Continual observation of clinical and immunological parameters, in particular of salivary IgA, in tonsillectomised children. Arch Otorhinolaryngol 1980;226:73-84.

${ }^{4}$ Ogra PL. Effect of tonsillectomy and adenoidectomy on nasopharyngeal antibody response to poliovirus. $N$ Engl f $\mathrm{Med} 1971 ; 284: 59-64$

(Accepted 22 December 1983)

University Hospital of Tromso, N-9012 Tromsø, Norway

BJØRN-ERIK KRISTIANSEN, MD, senior registrar, department of microbiology

HANS ELVERLAND, MD, consultant, department of otorhinolaryngology

Institute of Medical Biology, University of Tromsa, School of Medicine, N-9000 Tromse, Norway

KRISTIAN HANNESTAD, MD, professor

Correspondence and requests for reprints to: Dr Bjørn-Erik Kristiansen.

\section{Transtracheal injection of saline in the investigation of pneumonia}

One problem when investigating pneumonia and lower respiratory tract infections is that sputum may not be obtainable, even after physiotherapy. In recent studies $50-60 \%$ of patients could not produce sputum for examination. ${ }^{1-3}$ In such cases transtracheal aspiration, bronchoscopy, or percutaneous lung aspiration may be used to obtain lower respiratory secretion. ${ }^{4}$ Many clinicians, however, particularly in Britain, are unhappy about using such invasive techniques, especially for the investigation of community acquired pneumonia. We have investigated prospectively the usefulness of transtracheal saline injection for aiding the collection of lower respiratory tract secretions in patients with community acquired pneumonia.

\section{Patients, methods, and results}

Over 13 months 127 consecutive adults admitted to our hospital with community acquired pneumonia were included in a detailed clinical and laboratory study of the causes and outcome of pneumonia. ${ }^{5}$ An attempt was made to collect sputum from all patients on admission by voluntary coughing, usually with the aid of a physiotherapist. When sputum was unobtainable a transtracheal injection of saline was performed by the following method. The procedure was explained to the patient, who lay back at about $45^{\circ}$ in bed and extended the neck. The cricothyroid membrane was identified and the overlying skin cleaned. A 19 gauge needle attached to a $5 \mathrm{ml}$ syringe filled with sterile isotonic saline was inserted through the membrane into the tracheal lumen. Air was withdrawn to confirm placement and then the contents of the syringe instilled briskly into the trachea and the needle withdrawn immediately. Invariably the patient sat forward, coughed deeply, and produced a specimen of lower respiratory tract secretions into a wide mouthed specimen container. Premedication and local anaesthesia were not used, the whole procedure taking less than one minute.

Of the 127 patients studied, $70(55 \%)$ produced sputum specimens. Forty two of the remaining 57 patients were given a transtracheal injection of saline, and all except one produced specimens of lower respiratory tract secretions. Examination of these specimens by culture, and by countercurrent immunoelectrophoresis for pneumococcal antigen, ${ }^{4}$ yielded evidence of pneumococcal infection in 26 patients (table). Supporting evidence of pneumococcal infection was available for 23 of these patients by the culture of Streptococcus pneumoniae from serum (five cases) and/or by the detection of pneumococcal antigen in serum (six cases), pleural fluid (three cases), lung tissue (three cases), and/or concentrated urine ( 20 cases). Thirteen patients had no pathogens isolated on culture and no pneumococcal antigen detected in their lower 